\title{
ANALYSIS OF INCIDENCES OF COLLAPSED BUILDINGS IN LAGOS METROPOLIS, NIGERIA
}

\author{
Ayotunde Olawande ONI \\ Department of Estate Management, College of Science \& Technology, Covenant University, \\ Ota, Nigeria \\ E-mail:wandeoni@yahoo.com, telephone: 234-8023122014
}

Received 30 March 2009; accepted 26 April 2010

\begin{abstract}
Building collapses in Lagos metropolis have become worrisome to residents, developers, and Government. This study examined the incidences of collapsed buildings in Lagos metropolis over a thirty-year period. Time series analysis was carried out to determine the past and predict direction of the future occurrences. In addition, a process of inference from reports on investigations of past occurrences was adopted to establish causes of building collapses in the study area. Spatial analysis of the collapses showed high concentration in swampy terrain that was reclaimed in the past. The study recommends, amongst other things, comprehensive investigation of the geophysical characteristics of the affected locations towards finding lasting solution to the menace.
\end{abstract}

KEYWORDS: Collapsed building; Disaster; Distressed building; Spatial analysis; Lagos; Nigeria

\section{INTRODUCTION}

Buildings are expected to have certain characteristics that make them attractive for many uses which may be residential, commercial, institutional, educational, and industrial to meet people's daily needs. Characteristics of a good building include provision of security, safety to lives and properties, convenience, in addition to social, psychological and economic satisfactions derived by occupiers. In some cases, buildings that are expected to meet the people's daily needs have become source of great concerns to occupiers, owners, developers, governments, and physical development planning authorities, consequent upon their incessant failure and collapses.

Building failure, according to Ayininuola and Olalusi (2004), is an unacceptable difference between expected and observed performance of building components. They identified two types of failure in building, which are cosmetic and structural types. Cosmetic failure occurs when something has been added to or subtracted from the building, thus affecting the structures' outlooks. On the other hand, structural failure affects both the outlook and structural stability of the building.

Roddis (1993) considered failure as occurring in building components when they can no longer be relied upon to fulfill their principal functions. He distinguished between defect and failure in buildings. Defect is deflection in a building causing certain amount of cracking or distortion while excessive deflection that results in serious damage to partitions, ceilings and floor finishes is referred to as building failure. A distressed building exhibits defects 
in its components, noticeable as weakened foundation, cracks in floors, walls, and roofs. These more often signify overall defects in the structural stability of buildings which, if not checked, result in progressive collapse.

Failure, according to Wardhana and Hadipriono (2003), is the incapacity of a constructed facility or its components to perform as specified in the design and construction requirements. Building collapse is therefore the failure of all or a substantial part of a building, where full or partial replacement may be needed. Citing the collapse of the World Trade Centre tower as the most infamous paradigm, Bažant and Verdure (2006) described progressive collapse as a failure mode of great concern for tall building subjected to fire, internal explosions, external blast, impact, earthquake and foundation movements, and also typical of building demolitions.

According to Wardhana and Hadipriono (2003), collapse and distress are subsets of failure in a building. In terms of functionality, collapse occurs when the entire or a substantial part of a structure comes down thereby losing the ability to perform its function. Building collapse may be classified as total and partial collapses. Total collapse implies that several primary structural members of a building have fallen down completely while partial collapse suggests a condition where only some of the primary structural members of the building components have fallen down. Distress is the un-serviceability of a structure or its components that may or may not result in a collapse, and refers to a particular condition of the structure which has undergone some deformations without losing the whole structural integration.

Incidences of distressed or collapsed buildings are global phenomenon and are not limited to Nigeria. At the international level, a number of building collapses were reported - Ronan Point apartments collapse in U. K. when kitchen gas exploded on the 18th floor sending a 25-storey building to the ground in 1968; the 2000 Commonwealth Avenue Tower collapse in Boston, 1971; The Civic Center of Pavia in 1989; and collapse of Murrah Federal Building in Oklahoma City in 1995, where airblast pressure caused the collapse of few lower floors while the upper floors failed by progressive collapse. In year 2000, a four-storey commercial building at 14th and 2nd Avenue in Brooklyn, USA, collapsed and vacant building at 124th Street in the north of Manhattan in New York the partially collapsed in 2007. Similarly, a five-storey vacant apartment building in Manhattan that earlier appeared to be falling apart for months finally collapsed on March 4, 2008.

Other notable collapses include an uncompleted building that showed sign of breaking up in central Nairobi, Kenya collapsed in 2006; an apartment building in downtown Baku, Azerbaijan on August 28, 2007; and a twelve-storey apartment building in northern Egyptian port city of Alexandria, Egypt on December 19, 2007; while on 29 March, 2008 in Luanda, Angola, a six-storey police building collapsed with detainees and other people trapped and injured. In May 2008, a wall collapsed at a building site in Farooq Nagar, the suburbs of New Delhi, India; and hotel building consisted of a basement plus three upper floors located opposite Ahmedabad main railway station, Kalupur, India on February 3, 2008 (Heinle and Leonhardt, 1996; Binda et al., 1992; Levy and Salvadori, 1992; Bažant and Zhou, 2002; Pearson and Delatte, 2005; Bažant and Verdure, 2006; Adediji, 2006; Yussuf, 2006; Ismayilov, 2007; Islam, 2008).

The aim of this study was therefore to examine the incidences of collapsed buildings in Lagos metropolis with a view to determining their spatial distribution, including the number of such incidences from 1978 when building collapse was first reported up to 2007 when the study was carried out. The causes of and practical solutions for prevention of 
further occurrences are discussed alongside methods and materials used in analyzing the incidences.

\section{THE STUDY AREA}

According to Oni (2009), Lagos is the largest city in Nigeria with the estimates of its population varying considerably but generally range from 10 to 15.5 million people. It is the second largest city in Africa located at $6^{\circ} 34^{\prime} 60^{\prime \prime} \mathrm{N}, 3^{\circ} 19^{\prime} 59^{\prime \prime} \mathrm{E}$ along the West African coast. It is the former capital city of Nigeria replaced by Abuja on $12^{\text {th }}$ December, 1991 but remains the commercial centre of Nigeria. The city of Lagos is expected to hit 24.5 million-population mark and thus be among ten most populous cities in the world by the year 2015 .
The Lagos metropolitan area spreads over much of Lagos State, which itself covers an area of approximately 3,345 square kilometres. It is located on four principal islands and adjacent parts of mainland. The islands are connected to each other and to the mainland by bridges and landfills, with major sections of the metropolis that include the old city serving as the commercial district on western Lagos Island. Ikoyi Island is situated east of Lagos Island and joined to it by a landfill; while Apapa is the chief port district located on the mainland. Other components of the metropolis are Victoria Island, industrialized Iddo Island consisting of mainland suburbs, Ebute Meta, Yaba, Surulere, Ajegunle, Shomolu, Agege, Mushin and Ikeja (Oni, 2008).

Figure 1 is the map of Lagos metropolis showing the component Local Government Council Areas.

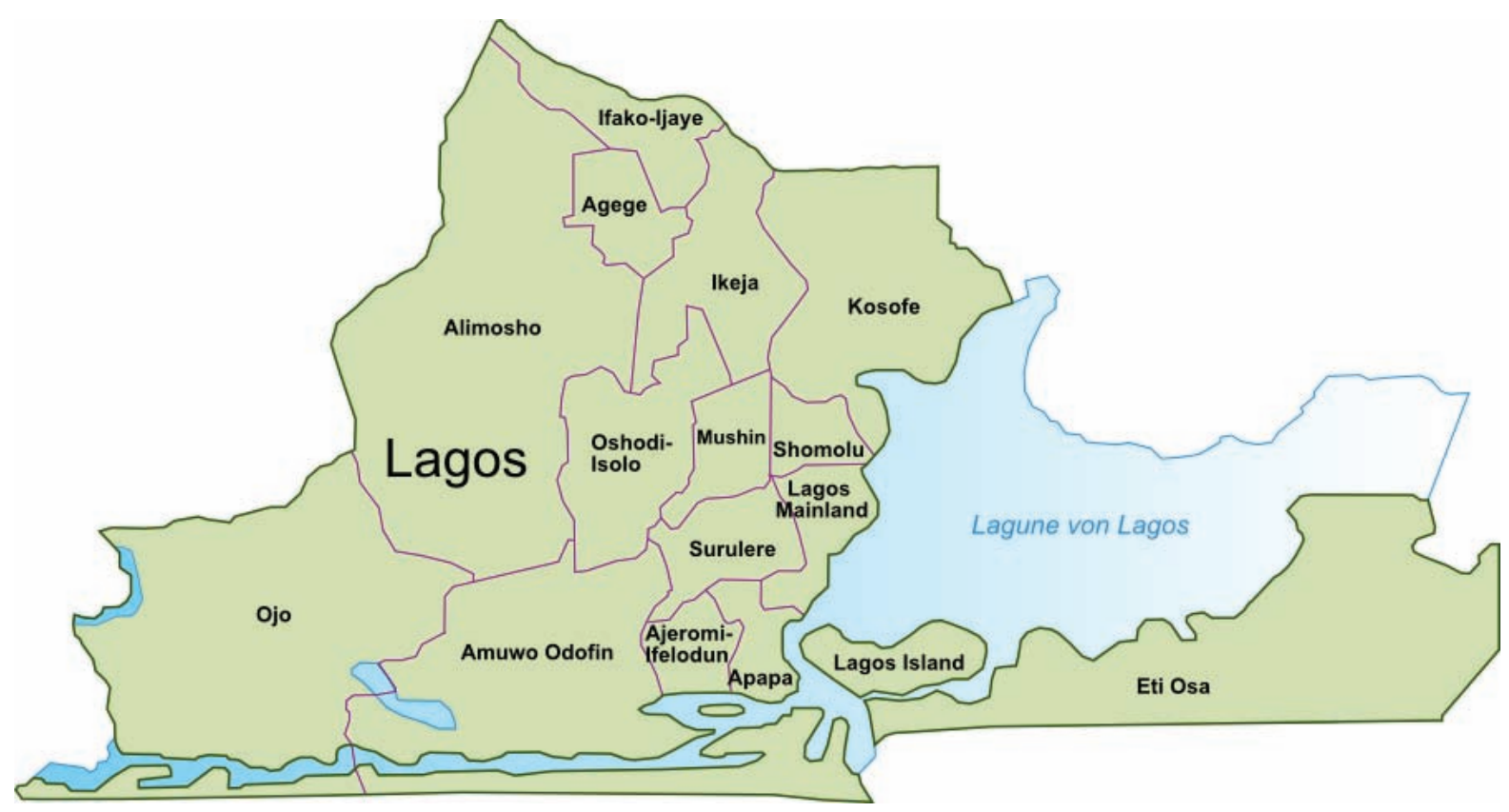

Figure 1. Map of Lagos metropolis showing the local government areas Source: Bohr (2006) 
The study area has witnessed rapid growth in demand for all types of properties, especially during the era of economic boom of the 1980s. Developers and owners attempted to meet shortage in supply of properties through direct construction of new structures or by redevelopment of existing ones. In meeting soaring demand for properties, many residential properties approved for one storey were converted to high rise structures (Adediji, 2006). The rush for investment in real estate during the period partly accounted for great activities in the construction period, with many construction activities lacking proper monitoring.

\section{MATERIALS AND METHODS}

The attempt was to examine the incidences of building collapse in Lagos metropolis, with a view to determining the spatial distribution of the incidences and predict the trend. In doing so, data were obtained mainly from the primary and secondary sources. Secondary sources included data from reports of investigation committees set up by the Lagos State Government. This was in addition to the number of incidences, addresses and descriptions of affected buildings obtained from journals, and Lagos State Physical Planning and Development Authority; while primary source included records personally obtained on recent incidences.

In examining the incidences of collapsed buildings in the study area, data were collected on a thirty-year period. This involved a process of inference which included an analysis of the reports of investigations of the incidences over the period to uncover the causes of collapse and distressed buildings for possible advice on policy formulation in the study area. Also, the map of the study area was used to depict the spatial distribution of the incidences, and equation for predicting future occurrences was derived with the aid of Microsoft Excel ${ }^{\circledR}$ software package.
In addition, trend analysis with the aid of the software package (selecting the linear trend option) was carried out to determine the behavior of past incidences and predict direction to which the incidences will move in future. The resulting figures were consequently illustrated in graph.

\section{FACTORS RESPONSIBLE FOR THE OCCURRENCES OF BUILDING COLLAPSE IN NIGERIA}

In general terms, earlier studies have identified a number of factors that are responsible for building collapse in Nigeria. Yussuf (2006) classified the causes as physical factors, ecological status of the site, composition of technical components, social factors, economic factors, engineering factors, human factors, government policies, and political factor.

Hall (1984) ascribed faulty design, faulty execution of work, and use of faulty materials as major causes of structural failures; while Merritt and Ambrose (1989) were of the opinion that overturning of structures due to heavy wind loads, sliding of structures due to high wind, roof uplift or sliding, and building sway due to lateral loads were major factors of failures in buildings. Oyewande (1992) identified additional factors responsible for building collapses in Nigeria; these are design faults, faults on construction site and product failure, with design fault contributing most significantly to such collapses. Other studies by Akinpelu (2002), and Richards (2002) found that structural failures, environmental changes, natural and human-induced hazards, improper presentation and interpretation in design, deterioration of reinforced concrete occurring as a result of corrosion of reinforcement caused by carbonation and chloride ingress, cracking caused by overloading, subsidence or basic design faults, and construction defects are causes of building collapses. 
Ayininuola and Olalusi (2004) opined that as a result of high cost of modern building materials, use of local methods of construction were employed without design codes. The compositions of the construction methods included structural slabs and all-round lintels of hollow sandcrete blocks of low compressive strength that sometimes resulted in sagging of slabs and crushing of blocks underneath the slabs. In addition, Ahmad (2004) found additional causes different from earlier studies: these are fungus stain and harmful growth, erosion of mortar joints, defective plastered rendering, cracking and leaning of walls, defective rainwater goods, decayed floor boards, insect or termite attack, dampness and penetration through walls, and unstable foundations.

According to Ozerdem (1999) and Global Corruption Report (2005), corruption has been identified as one factor that has caused high cost of building materials and reduction in standard of construction works in developing economy. They stated that corruption may be at different stages, namely, at contract award, planning and design stage, construction stage, and when the building is completed. It may take different forms like bribery, deception and collusion the end products of which are lowering of construction standard, increasing cost of repair and maintenance, defects in building that may not be discovered until eventually collapse.

\section{INCIDENCES OF COLLAPSED BUILDINGS IN LAGOS METROPOLIS}

In Lagos metropolis, many buildings have collapsed and only those that were critical have been reported while many others were unreported probably due to their obscurity to Press and Government officials. The first major incidence of collapsed buildings was recorded in 1978 and the incidences have become incessant since then.

Although incidence of earthquake has never been reported in the study area, the blast in 2002 of high caliber bombs stored in underground bunkers within the Ikeja military cantonment might have created features of an earthquake prone area, which Lewis (2003) described as structural inadequacy exposed by failure and collapse of buildings.

Details of the occurrences, locations, descriptions of the properties, and causes of the collapses are shown in Table 1.

Table 1. Details of collapsed buildings in Lagos metropolis (1978-2007)

\begin{tabular}{|c|c|c|c|c|c|c|}
\hline $\mathrm{SN}$ & Year & Occurrences & Location/Address & Description & Cause* & Remarks \\
\hline 1 & 1978 & 1 & $\begin{array}{l}\text { Western Avenue, } \\
\text { Surulere }\end{array}$ & 3 -storey building & Structural defects & - \\
\hline 2 & 1979 & 0 & - & - & Structural defects & - \\
\hline 3 & 1980 & 0 & - & - & Structural defects & - \\
\hline 4 & 1981 & 0 & - & - & Structural defects & - \\
\hline 5 & 1982 & 0 & - & - & Structural defects & - \\
\hline \multirow[t]{3}{*}{6} & 1983 & 3 & Lewis St. Lagos Island & 4-storey building & Structural defects & - \\
\hline & & & Iponri & 2-storey building & Structural defects & - \\
\hline & & & Ojuelegba Road & 2-storey building & Structural defects & - \\
\hline 7 & 1984 & 1 & Beecroft Street & 2-storey building & Structural defects & $\begin{array}{l}- \\
\text { (Continued) }\end{array}$ \\
\hline
\end{tabular}




\begin{tabular}{|c|c|c|c|c|c|c|}
\hline $\mathrm{SN}$ & Year & Occurrences & Location/Address & Description & Cause* & Remarks \\
\hline \multicolumn{7}{|c|}{ (Continued) } \\
\hline \multirow[t]{7}{*}{8} & 1985 & 7 & Western Avenue, Iponri & 4-storey building & Structural defects & - \\
\hline & & & Lagos Island & 3-storey building & Structural defects & - \\
\hline & & & Ojuelegba Road & 2 -storey building & Poor materials & - \\
\hline & & & Allen Avenue, Ikeja & $\begin{array}{l}\text { Uncompleted four- } \\
\text { storey }\end{array}$ & Structural defects & - \\
\hline & & & $\begin{array}{l}\text { Bereka Lane, Lagos } \\
\text { Island }\end{array}$ & $\begin{array}{l}\mathrm{U} / \mathrm{C} \text { two-storey } \\
\text { building }\end{array}$ & Structural defects & - \\
\hline & & & Adeniji Adele St, & 3 -storey building & Structural defects & - \\
\hline & & & $\begin{array}{l}\text { Ojuelegba Junction, } \\
\text { Ayilara }\end{array}$ & 2 -storey building & Improper design & - \\
\hline \multirow[t]{5}{*}{9} & 1986 & 5 & Western Avenue, Iponri & $\begin{array}{l}\text { U/C } 6 \text {-storey } \\
\text { building }\end{array}$ & Poor materials & - \\
\hline & & & Usabi, Maryland & $\begin{array}{l}\text { U/C 3-storey } \\
\text { building }\end{array}$ & Poor materials & - \\
\hline & & & Orile- Iganmu & $\begin{array}{l}\text { U/C 3-storey } \\
\text { building }\end{array}$ & Poor materials & - \\
\hline & & & $\begin{array}{l}\text { Berkley Lane, Island } \\
\text { Island }\end{array}$ & $\begin{array}{l}\text { U/C 2-storey } \\
\text { building }\end{array}$ & Poor materials & - \\
\hline & & & Idunsagbe Lane, Island & $\begin{array}{l}\text { U/C 3-storey } \\
\text { building }\end{array}$ & Poor materials & - \\
\hline \multirow[t]{7}{*}{10} & 1987 & 7 & Agege, & 2 -storey building & Structural defects & - \\
\hline & & & Ikorodu Rd, Somolu LG & 3-storey building & Structural defects & - \\
\hline & & & Akinade Village, Ikeja & 2 -storey building & Structural defects & - \\
\hline & & & Ikorodu Rd Ketu & 3-storey building & Structural defects & - \\
\hline & & & $\begin{array}{l}20 \text { Idunsagbe Lane, } \\
\text { Island }\end{array}$ & 4-storey building & Structural defects & - \\
\hline & & & Idumagbo Area, Island & 3 -storey building & Structural defects & - \\
\hline & & & Agege Road & 3 -storey building & Structural defects & - \\
\hline 11 & 1988 & 0 & - & - & - & - \\
\hline \multirow[t]{4}{*}{12} & 1989 & 4 & Adeniji Adele, Island & 4-storey building & Dilapidation & - \\
\hline & & & $\begin{array}{l}\text { Akinwumi St, Mende, } \\
\text { Ikeja }\end{array}$ & $\begin{array}{l}8 \text {-storey Hotel } \\
\text { building }\end{array}$ & Structural defects & - \\
\hline & & & Idumota, Island & $\begin{array}{l}\text { 6-storey Royal } \\
\text { Hotel }\end{array}$ & Structural defects & - \\
\hline & & & Beecroft Lane, Island & School building & Structural defects & - \\
\hline \multirow[t]{3}{*}{13} & 1990 & 3 & Idi- oro, Mushin & $\begin{array}{l}\text { U/C 3-storey } \\
\text { building }\end{array}$ & Structural defects & - \\
\hline & & & Idumota, Island & $\begin{array}{l}\text { U/C 3-storey } \\
\text { building }\end{array}$ & Structural defects & - \\
\hline & & & Idumagbo, Island & 4-storey office block & Structural defects & - \\
\hline \multirow[t]{3}{*}{14} & 1991 & 2 & $\begin{array}{l}\text { Moyosore Close, } \\
\text { Gbagada, Somolu }\end{array}$ & $\begin{array}{l}\text { U/C 3-storey } \\
\text { building }\end{array}$ & Structural defects & - \\
\hline & & & $\begin{array}{l}\text { UNILAG, Faculty of } \\
\text { Education }\end{array}$ & Lecture Hall, & Structural defects & - \\
\hline & & & & & & (Continued) \\
\hline
\end{tabular}




\begin{tabular}{|c|c|c|c|c|c|c|}
\hline $\mathrm{SN}$ & Year & Occurrences & Location/Address & Description & Cause* & Remarks \\
\hline \multicolumn{7}{|c|}{ (Continued) } \\
\hline \multirow[t]{3}{*}{15} & 1992 & 3 & $\begin{array}{l}\text { Hawley Road, Sabo, } \\
\text { Yaba }\end{array}$ & 2 -storey building & Structural defects & - \\
\hline & & & Oyadiran Estate, Yaba & 2 -storey building & Structural defects & - \\
\hline & & & Alafia Street, Mushin & $\begin{array}{l}\text { U/C 3-storey } \\
\text { building }\end{array}$ & Poor materials & - \\
\hline 16 & 1993 & 1 & Adeniyi Close, Bariga & Block of 4 No. flats & Structural defects & - \\
\hline \multirow[t]{6}{*}{17} & 1994 & 6 & Airport Road, Oshodi & $\begin{array}{l}\text { U/C 3-storey office } \\
\text { block }\end{array}$ & Poor materials & - \\
\hline & & & Okesuna Road, Island & Hotel building & Structural defects & - \\
\hline & & & Idumagbo Area, Island & $\begin{array}{l}\text { U/C 3-storey } \\
\text { building }\end{array}$ & Structural defects & - \\
\hline & & & Ajah, Etiosa & Estate building & Structural defects & - \\
\hline & & & Onikan, Island & 3rd Axial Bridge & Structural defect & - \\
\hline & & & Airport Road, Oshodi & 3 -storey building & Structural defects & - \\
\hline \multirow[t]{8}{*}{18} & 1995 & 8 & Ajao Estate, & $\begin{array}{l}\text { U/C 4-storey } \\
\text { building }\end{array}$ & Poor materials & - \\
\hline & & & Wale Ajose St, Mende, & $\begin{array}{l}\text { U/C } 6 \text {-storey } \\
\text { building }\end{array}$ & Structural defects & - \\
\hline & & & Maryland, Ikeja & $\begin{array}{l}\text { U/C 3-storey office } \\
\text { block }\end{array}$ & Structural defects & - \\
\hline & & & Ogba, Ikeja & $\begin{array}{l}\mathrm{U} / \mathrm{C} 2 \text {-storey place } \\
\text { of worship }\end{array}$ & Structural defects & - \\
\hline & & & Agege & 2-storey building & Structural defects & - \\
\hline & & & Ikorodu Road, Maryland & $\begin{array}{l}\text { U/C } 5 \text {-storey } \\
\text { building }\end{array}$ & Poor material & - \\
\hline & & & Agege & 3 -storey building & Structural defects & - \\
\hline & & & Iponri & $\begin{array}{l}\mathrm{U} / \mathrm{C} 2 \text {-storey } \\
\text { building }\end{array}$ & Structural defects & - \\
\hline \multirow[t]{3}{*}{19} & 1996 & 3 & Iponri & 4-storey building & Structural defects & - \\
\hline & & & Alapere, Ketu & $\begin{array}{l}\text { Aiyedere Primary } \\
\text { School }\end{array}$ & Structural defects & - \\
\hline & & & $\begin{array}{l}\text { 20, Olowookere St., } \\
\text { Oshodi }\end{array}$ & $\begin{array}{l}\mathrm{U} / \mathrm{C} 2 \text {-storey } \\
\text { building }\end{array}$ & Structural defects & - \\
\hline 20 & 1997 & 0 & - & - & - & - \\
\hline \multirow[t]{2}{*}{21} & 1998 & 2 & Mafoluku, Oshodi & $\begin{array}{l}\mathrm{U} / \mathrm{C} \text { Church } \\
\text { building }\end{array}$ & Structural defects & - \\
\hline & & & Igbobi, Somolu & $\begin{array}{l}\text { U/C 3-storey } \\
\text { building }\end{array}$ & Structural defects & - \\
\hline \multirow[t]{6}{*}{22} & 1999 & 5 & Olowookere, Oshodi & Apostolic Church & Structural defects & - \\
\hline & & & Oke- Igbala, Mushin & 3-storey building & Structural defects & - \\
\hline & & & Idusagbe Lane, Island & 2-storey building & Structural defects & - \\
\hline & & & Idumota Area, Island & $\begin{array}{l}\text { U/C 3-storey } \\
\text { building }\end{array}$ & Poor materials & - \\
\hline & & & Ikota, Ajah & Eleganza building & Structural defects & - \\
\hline & & & & & & (Continued) \\
\hline
\end{tabular}




\begin{tabular}{|c|c|c|c|c|c|c|}
\hline $\mathrm{SN}$ & Year & Occurrences & Location/Address & Description & Cause* & Remarks \\
\hline \multicolumn{7}{|c|}{ (Continued) } \\
\hline \multirow[t]{7}{*}{23} & \multirow[t]{7}{*}{$2000^{*}$} & \multirow[t]{7}{*}{7} & $\begin{array}{l}\text { 3/13 Thomas Drive, } \\
\text { Bariga }\end{array}$ & $\begin{array}{l}\text { St. Dennis Catholic } \\
\text { Church }\end{array}$ & $\begin{array}{l}\text { Pressure on } \\
\text { uncompleted } \\
\text { structure. } \\
\text { Structural defects }\end{array}$ & $\begin{array}{l}120 \text { people } \\
\text { trapped and } \\
\text { injured; three } \\
\text { death recorded }\end{array}$ \\
\hline & & & $\begin{array}{l}\text { 14, Semi Sarumi Street, } \\
\text { Itire }\end{array}$ & $\begin{array}{l}\text { Residential } \\
\text { property being } \\
\text { reconstructed, first } \\
\text { floor slab collapsed }\end{array}$ & $\begin{array}{l}\text { Deterioration of } \\
\text { existing building; } \\
\text { improper design }\end{array}$ & $\begin{array}{l}\text { One woman } \\
\text { died }\end{array}$ \\
\hline & & & $\begin{array}{l}\text { State High School, } \\
\text { Alimosho }\end{array}$ & School wall fence & $\begin{array}{l}\text { Weak and un- } \\
\text { braced wall }\end{array}$ & $\begin{array}{l}3 \text { students } \\
\text { injured, one } \\
\text { died }\end{array}$ \\
\hline & & & $\begin{array}{l}\text { 56A Adeniji Adele } \\
\text { Street, Island }\end{array}$ & $\begin{array}{l}\text { Part of a } 4 \text {-strorey } \\
\text { building of about } \\
25 \text { rooms }\end{array}$ & $\begin{array}{l}\text { Contravention of } \\
\text { planning approval }\end{array}$ & $\begin{array}{l}\text { No death or } \\
\text { injury }\end{array}$ \\
\hline & & & $\begin{array}{l}10-12, \text { Suene Street, } \\
\text { Surulere }\end{array}$ & $\begin{array}{l}\text { 4-storey twin } \\
\text { building }\end{array}$ & $\begin{array}{l}\text { Re- partitioning } \\
\text { of parts of } \\
\text { the building. } \\
\text { Structural defects }\end{array}$ & $\begin{array}{l}\text { Two people } \\
\text { died. }\end{array}$ \\
\hline & & & Sanmi Street, Itire & Not available & & - \\
\hline & & & $\begin{array}{l}\text { Folami Primary School, } \\
\text { Atunrase Street, } \\
\text { Surulere }\end{array}$ & Perimeter wall & Structural defects & $\begin{array}{l}\text { Deaths and } \\
\text { injuries }\end{array}$ \\
\hline \multirow[t]{2}{*}{24} & \multirow[t]{2}{*}{$2001^{*}$} & \multirow[t]{2}{*}{2} & Igbosere Street, Island & $\begin{array}{l}\text { Uncompleted } \\
\text { 3-storey building }\end{array}$ & Structural defects & - \\
\hline & & & $\begin{array}{l}\text { Idusagbe Lane, Lagos } \\
\text { Island }\end{array}$ & $\begin{array}{l}\text { Uncompleted } \\
\text { 4-storey building }\end{array}$ & Structural defects & - \\
\hline \multirow[t]{5}{*}{25} & \multirow[t]{5}{*}{$2002^{*}$} & \multirow[t]{5}{*}{6} & Agege Road & 2 -storey building & Structural defects & \multirow{5}{*}{$\begin{array}{l}\text { Note: Bomb } \\
\text { blast occurred. } \\
\text { Many roofs, } \\
\text { collapsed } \\
\text { but were } \\
\text { unreported }\end{array}$} \\
\hline & & & Allen Avenue, Ikeja & 3-storey building & Structural defects & \\
\hline & & & $\begin{array}{l}\text { Isiaka Street, off Agege } \\
\text { Road }\end{array}$ & 2 -storey building & Structural defects & \\
\hline & & & $\begin{array}{l}10 \text { Jones Street, Ebute } \\
\text { Metta West } \\
8, \text { Abeokuta Street, } \\
\text { Ebute Metta }\end{array}$ & 3 -storey building & $\begin{array}{l}\text { Dilapidation. } \\
\text { Structural defects } \\
\text { Structural defects }\end{array}$ & \\
\hline & & & 49, Olonode Street, Yaba & 2 -storey building & Structural defects & \\
\hline \multirow[t]{4}{*}{26} & \multirow[t]{4}{*}{$2003^{*}$} & \multirow[t]{4}{*}{6} & $\begin{array}{l}50 \text { Willoughby Street, } \\
\text { Ebute Metta }\end{array}$ & 1-storey building & Structural defects & - \\
\hline & & & $\begin{array}{l}28 \text { Idumagbo Avenue, } \\
\text { Island }\end{array}$ & 3-storey building & $\begin{array}{l}\text { Explosion } \\
\text { from storage } \\
\text { of pyrotechnic } \\
\text { devices used in fire } \\
\text { works }\end{array}$ & $\begin{array}{l}30 \text { people died, } \\
60 \text { injured }\end{array}$ \\
\hline & & & $\begin{array}{l}\text { Pedro Street, near } \\
\text { Idumagbo Avenue, Lagos } \\
\text { Island }\end{array}$ & 3 -storey building & $\begin{array}{l}\text { Impact of the } \\
\text { explosion at } 28 \\
\text { Idumagbo Avenue }\end{array}$ & - \\
\hline & & & & & & (Continued) \\
\hline
\end{tabular}




\begin{tabular}{|c|c|c|c|c|c|c|}
\hline $\mathrm{SN}$ & Year & Occurrences & Location/Address & Description & Cause* & Remarks \\
\hline \multicolumn{7}{|c|}{ (Continued) } \\
\hline & & & Bereka Lane, Lagos & $\begin{array}{l}\text { Uncompleted } \\
\text { 3-storey building }\end{array}$ & Structural defects & - \\
\hline & & & Adeniji Adele Road & $\begin{array}{l}\text { Uncompleted } \\
\text { 4-storey building }\end{array}$ & Structural defects & - \\
\hline & & & Ojuelegba Road & 3 -storey building & $\begin{array}{l}\text { Construction } \\
\text { defects }\end{array}$ & - \\
\hline \multirow[t]{3}{*}{27} & $2004^{*}$ & 3 & $\begin{array}{l}\text { 22, Markurdi Street, } \\
\text { Ebute Metta }\end{array}$ & 3-storey building & Structural defects & - \\
\hline & & & Ilasamaja Mushin & $\begin{array}{l}\text { Uncompleted } \\
2 \text {-storey building }\end{array}$ & Poor materials & - \\
\hline & & & $\begin{array}{l}\text { 10, Elias Street, Lagos } \\
\text { Island }\end{array}$ & $\begin{array}{l}\text { Partial collapse of } \\
2 \text {-storey structure }\end{array}$ & $\begin{array}{l}\text { Dilapidation, lack } \\
\text { of maintenance }\end{array}$ & - \\
\hline \multirow[t]{6}{*}{28} & $2005^{*}$ & 6 & $\begin{array}{l}\text { 40, Market Street, } \\
\text { Shomolu }\end{array}$ & $\begin{array}{l}\text { Partial collapse of } \\
\text { decking of } 2 \text {-storey } \\
\text { building }\end{array}$ & Structural defects & - \\
\hline & & & $\begin{array}{l}\text { Kodesho/Bank-Anthony } \\
\text { Way }\end{array}$ & 2 -storey building & Structural defects & - \\
\hline & & & $\begin{array}{l}\text { Adeniji Adele, Lagos } \\
\text { Island }\end{array}$ & $\begin{array}{l}\text { Uncompleted } \\
\text { 3-storey building }\end{array}$ & Structural defects & - \\
\hline & & & $\begin{array}{l}\text { 6, Princess Street, Lagos } \\
\text { Island }\end{array}$ & 3 -storey building & $\begin{array}{l}\text { Poor construction } \\
\text { material }\end{array}$ & - \\
\hline & & & Iponri, Lagos & Wall fence & Structural defects & - \\
\hline & & & Mende, Maryland, Ikeja & $\begin{array}{l}\text { Uncompleted } \\
2 \text {-storey building }\end{array}$ & Structural defects & - \\
\hline \multirow[t]{7}{*}{29} & $2006^{*}$ & 7 & $\begin{array}{l}\text { 53, Cemetery Road, } \\
\text { Amukoko }\end{array}$ & 4 -storey building & Structural defects & - \\
\hline & & & Ijora, Ajegunle & 3-storey building & Structural defects & - \\
\hline & & & $\begin{array}{l}\text { NIDB Building, Broad } \\
\text { Street, Lagos Island }\end{array}$ & High rise building & Fire incidence & - \\
\hline & & & $\begin{array}{l}\text { 71, Ibadan Street, Ebute } \\
\text { Metta West }\end{array}$ & $\begin{array}{l}3 \text {-storey building } \\
\text { with Penthouse }\end{array}$ & Structural defects & - \\
\hline & & & $\begin{array}{l}\text { 42, Ibadan Street, Ebute } \\
\text { Metta East }\end{array}$ & 3 -storey building & Structural defects & - \\
\hline & & & $\begin{array}{l}\text { 1, Murtala Muhammed } \\
\text { Airport Road Oshodi }\end{array}$ & 2 -storey building & Structural defects & - \\
\hline & & & $\begin{array}{l}\text { 6A Milverton Close, } \\
\text { Ikoyi }\end{array}$ & $\begin{array}{l}\text { collapse of car-park } \\
\text { beam }\end{array}$ & Dilapidation & - \\
\hline \multirow[t]{7}{*}{30} & $2007 *$ & 7 & $\begin{array}{l}\text { 118, Ojuelegba Road, } \\
\text { Surulere }\end{array}$ & 2 -storey building & Structural defects & - \\
\hline & & & $\begin{array}{l}\text { LASU-Iba Road, opposite } \\
\text { Rosellas }\end{array}$ & 2 -storey building & Structural defects & - \\
\hline & & & 48, Adams Street, Lagos & 3 -storey building & Structural defects & - \\
\hline & & & $\begin{array}{l}38, \text { Idumagbo Avenue, } \\
\text { Island }\end{array}$ & 3 -storey building & Structural defects & - \\
\hline & & & $\begin{array}{l}\text { 32B Egerton Lane, Oke } \\
\text { Arin }\end{array}$ & 4 -storey building & Structural defects & - \\
\hline & & & 71 Agoro Street, Lagos & 3 -storey building & Structural defects & - \\
\hline & & & $\begin{array}{l}8 \text { Ashaka Street, Abule- } \\
\text { Nla Ebute Metta }\end{array}$ & 2 -storey building & Structural defects & - \\
\hline
\end{tabular}

Source: Lagos state physical planning and development authority; * Report of standing committee on collapsed building in Lagos state 
From Table 1 a summary of the occurrences of collapsed buildings according to locations within the Local Government Council Areas is shown in Table 2.

Table 2. Distribution of collapsed buildings according to local government council areas in Lagos metropolis

\begin{tabular}{llll}
\hline S/N & Local government & Occurrences & Percentage \\
\hline 1 & Agege & 6 & 5.71 \\
2 & Ajeromi-Ifelodun & 2 & 1.90 \\
3 & Alimosho & 2 & 1.90 \\
4 & Apapa & 0 & 0 \\
5 & Etiosa & 3 & 2.86 \\
6 & Ifako-Ijaiye & 0 & 0 \\
7 & Ikeja & 11 & 10.48 \\
8 & Kosofe & 2 & 1.90 \\
9 & Lagos Mainland & 12 & 11.43 \\
10 & Lagos Island & 31 & 29.52 \\
11 & Mushin & 7 & 6.67 \\
12 & Oshodi & 7 & 6.67 \\
13 & Somolu & 7 & 6.67 \\
14 & Surulere & 15 & 14.29 \\
15 & Amuwo-Odofin & 0 & 0 \\
16 & Ojo & 0 & 0 \\
\hline Total & & 105 & 100.00 \\
\hline
\end{tabular}

\section{ANALYSIS AND DISCUSSION}

An examination of the spatial distribution of incidences of collapsed buildings in Lagos metropolis indicates that the occurrences are not evenly distributed; this is illustrated in Figure 1.

Figure 2 shows the spatial distribution of building collapses in the study area. Lagos Island recorded 31 (about 30\%); followed by Surulere, 15 (about 14\%); and Lagos Mainland, 12 (about 11\%), while no incidence was reported for Apapa, Ifako-Ijaiye, Amuwo-Odofin, and Ojo Local Government Areas of the study area. Many of the collapses were concentrated around swampy terrain not far from the Lagos Lagoon, where land was reclaimed for building purposes many years ago without strict planning approval process.

These data were however not normalized according to the Local Government Council Areas due to dearth of statistics on the standard and number of properties. The Government of Lagos State tried to carry out household and property survey in 2006 to determine the number of households and physical condition of properties

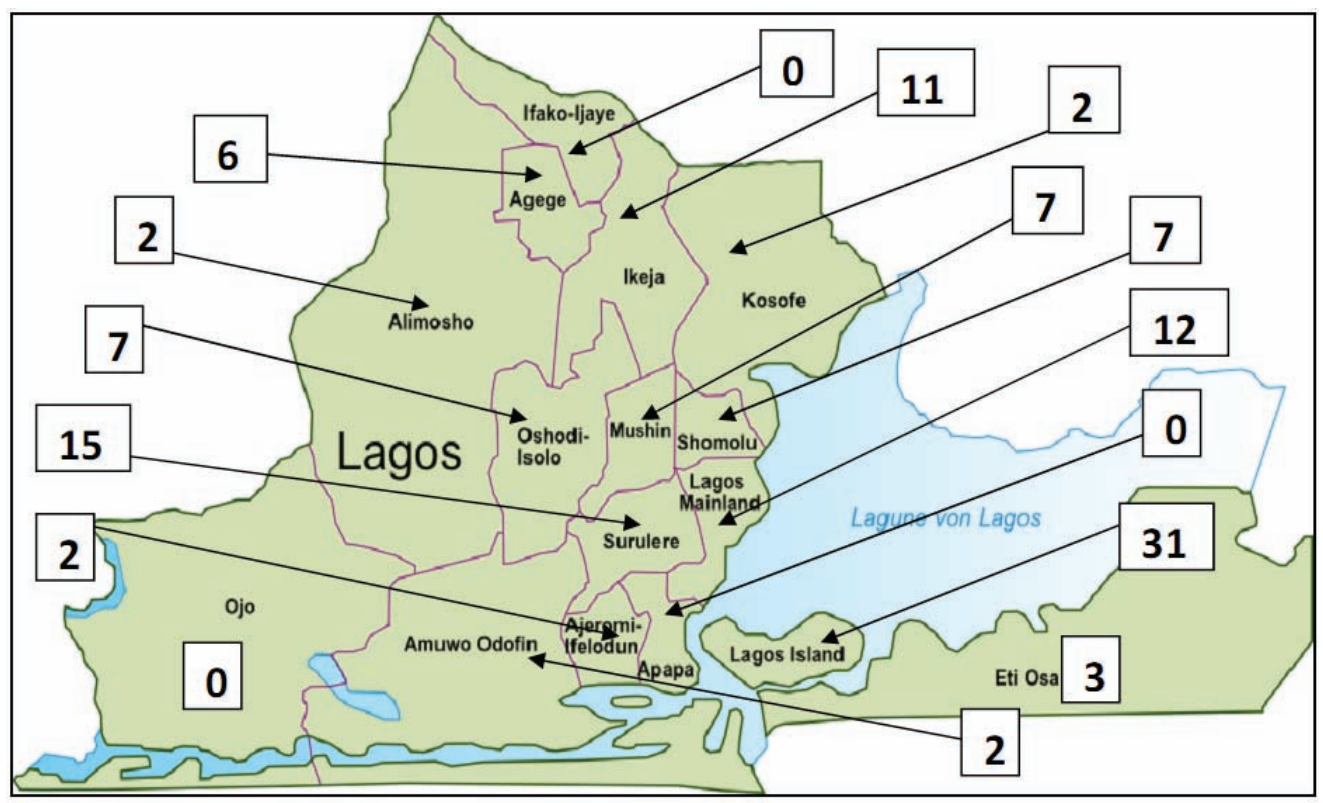

Figure 2. Map of Lagos metropolis showing spatial distribution of collapsed buildings 
in the State, little success was attained and the result has not been made public. This is one of the challenges that researchers face in creating complete data set of buildings collapses in an emerging economy like Nigeria. Nevertheless, the data are reliable and will assist Nigerian and non-Nigerian researchers. Consequently, in determining the trend in building collapses, the number of occurrences on annual basis shown in Table 3 was used.

Table 3. Details of incidences of building collapse in Lagos metropolis (1978-2007)

\begin{tabular}{|c|c|c|}
\hline $\mathrm{S} / \mathrm{N}$ & Year & Occurrences \\
\hline 1 & 1978 & 1 \\
\hline 2 & 1979 & 0 \\
\hline 3 & 1980 & 0 \\
\hline 4 & 1981 & 0 \\
\hline 5 & 1982 & 0 \\
\hline 6 & 1983 & 3 \\
\hline 7 & 1984 & 1 \\
\hline 8 & 1985 & 7 \\
\hline 9 & 1986 & 5 \\
\hline 10 & 1987 & 7 \\
\hline 11 & 1988 & 0 \\
\hline 12 & 1989 & 4 \\
\hline 13 & 1990 & 3 \\
\hline 14 & 1991 & 2 \\
\hline 15 & 1992 & 3 \\
\hline 16 & 1993 & 1 \\
\hline 17 & 1994 & 6 \\
\hline 18 & 1995 & 8 \\
\hline 19 & 1996 & 3 \\
\hline 20 & 1997 & 0 \\
\hline 21 & 1998 & 2 \\
\hline 22 & 1999 & 5 \\
\hline 23 & 2000 & 7 \\
\hline 24 & 2001 & 2 \\
\hline 25 & 2002 & 6 \\
\hline 26 & 2003 & 6 \\
\hline 27 & 2004 & 3 \\
\hline 28 & 2005 & 6 \\
\hline 29 & 2006 & 7 \\
\hline 30 & 2007 & 7 \\
\hline
\end{tabular}

The analysis of the data in Table 3 shows upward and downward movements in the number of building collapses in the study area from year to year over a thirty-year period, with the peak indicated for 1995 when eight occurrences were recorded as illustrated in Figure 3.

\section{RESEARCH FINDINGS}

By inference from earlier studies cited, the general causes of building collapsed were identified to include lack of design codes of building materials; faulty structural design; natural and human-made hazards; improper presentation and interpretation of design; deterioration of reinforced concrete; insects including termites and fungus attack, erosion of mortar joints, and defective plastering.

Specifically from this study, deductions from the reported incidences showed that causes of building collapses in Lagos metropolis include improper or bad design, construction defects, foundation failure, extraordinary loads, structural defects, dilapidation due to lack of maintenance, use of weak and substandard building materials, and storage of explosive devices. Out of one hundred and five collapses between 1978 and 2007, about 69\% were attributed to structural defects; $16 \%$ to poor quality materials; and $6 \%$ to defects in construction. Improper design caused about $4 \%$ of the collapses; dilapidation due to non-maintenance, $4 \%$; and storage of explosives, $1 \%$.

Furthermore, the study found that many of the occurrences were not investigated until year 2000 when the "Standing Committee on Collapsed Building in Lagos State" was set up by the Lagos State Government. The reports of the committee on few investigations since 2000 lacked in-depth analysis of the subject-matter and were prepared against the backdrop of shallow investigations to reveal actual causes, and only repeated the same causative factors over the years for every of the incidences. 


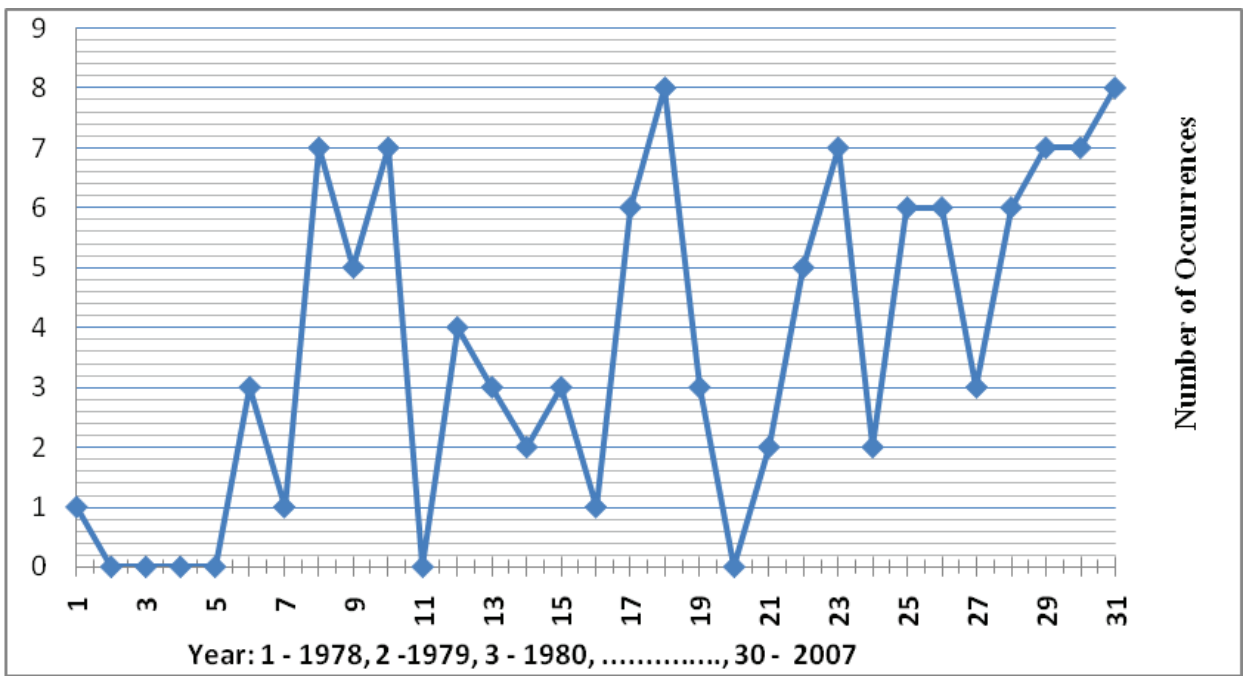

Figure 3. Trend in occurrences of building collapse in Lagos metropolis (1978-2007)

A perusal of the reports presented by the committee showed that opinion of members was suspect as they lacked the tools to carry out technical investigations. Reports on previous occurrences were slack in terms of technical format, expression and content that investigation of such magnitude demanded. The findings of the Committees did not discover the remote causes of the collapses and so could not assist government in policy formulation and implementation.

It was found that the annual average of four building collapses occurred within the thirty-year study period. However, an examination of the data from February 2002, when the incidence of bomb blast occurred in Ikeja, revealed that annual average over the period of five years (2002 to 2007) increased to seven. This is an indication that since the incidence of the bomb blast the annual average of building collapses has doubled. Also, the predictive linear trend equation showed that the incidences will increase from seven in 2007 to ten in year 2016. Total number of occurrences predicted for next ten years up to 2017 is ninety-one, indicating an average of nine per annum, all things being equal.
It was also found that high number of building collapses was recorded in parts of Lagos metropolis that were within swampy terrain reclaimed many years ago for building construction. Lagos Island, for instance, recorded average of one collapse every year, with Surulere and Lagos Mainland recording average of one collapse every two years in the past thirty years.

\section{RECOMMENDATIONS, POLICY IMPLICATIONS, AND CONCLUSION}

It is must be stated that every incidence is unique and must so be treated by adopting unique approach and thorough investigation to discover hidden causative factors for each occurrence. That structural defects have accounted for greatest number of collapses requires further investigation to ascertain the remote causes of such defects. High occurrences of collapsed buildings in Lagos Island, Surulere, and Lagos Mainland Local Government Council areas of the metropolis should not be treated as mere coincidence; they were swampy, sand-filled, and reclaimed terrains close to the Lagoon. Detailed study of the geophysical 
characteristics of the soils in all locations of the study area must be carried out urgently to establish their compositions and appropriate measures taken if the seemingly stunning prediction in this study is to be averted.

The impact of vibration from the Ikeja bomb blast which was felt at locations many kilometers away is a pointer to likely serious damage to the soil components and strata of locations within that radius. There is urgent need to determine its impact on the topography and stability of the earth crust. As stated earlier, although no incidence of earthquake has been reported in the study area, it is possible that a fault-line might have been created in the crust probably due to the impact of the vibrations from the explosion. To assume that the blast would not have caused serious damage yet undiscovered will be highly dangerous. By determining the impacts, locations that have propensity of collapsed buildings will be identified and precautionary measures to avoid loss of lives will be put in place and prevent unplanned management strategies often adopted during unexpected disasters.

Investigations into previous incidences were not thorough and incompetence on the part of members of the committee set up to find effective and reliable solutions to the incidences was glaring. Reports and findings of previous investigations were too elementary for practical purpose and intent to find lasting solution to the menace. Professionals related to structural, civil, and building engineering, soil characterization and testing should be consulted and appointed to serve as members of the investigation committee.

However, these tasks are enormous and capital intensive, requiring strong political will and enabling law which only the Federal and State Governments could successfully muster than individual property owners and researchers. The onus is therefore on the Federal and Lagos State Governments to commission aca- demics and researchers for further research into the incidences of building collapse, especially the impact of the bomb blast on stability of the building structures in the study area. Governments have powers to enact and enforce laws, it is therefore recommended that Building Codes be passed into law to enforce minimum standard of building materials, qualification of builders, and other issues relating thereto.

The Community Development Associations (CDAs) operate at the grass root and will play active role in stemming building collapses. This will be by identifying buildings within their communities that show signs of physical and structural defects and promptly report same to the Lagos State Physical Planning and Development Authority which must be established and charged with responsibility to enforce the building codes and implementation of the strategies for stemming incidences of building collapse in Lagos metropolis. The strategies should include auditing of buildings and physical developments including bridges in the study area to detect distress and avert collapse; establishment of physical development inspection and monitoring team composed of experts in building engineering and technology to monitor construction works and issue certificates of quality work at stages of development, members of such team must be above boards and not be the corrupt ones - practicing experts in building construction will serve this purpose; enforcement and ensuring compliance with approved design after due inspection; and testing of the soil on which the buildings are to be erected.

The academic and researchers must take interests in building survey to create complete data set of buildings, including their standard, structural stability, and state of repairs which must be updated regularly. The Nigeria Building and Road Research Institute should be empowered through provision of materials and 
fund to carry out research, especially in developing locally-made building materials to meet local requirements and specifications thereby preventing use of substandard materials. This will also go a long way at assisting Nigerian and non-Nigerian researchers in having access to such material and reduce incidences of building collapse to the minimum.

Lastly, it must be stated that compulsory acquisition of the sites and prosecution of supervising structural or civil engineers and owners of collapsed buildings as contemplated by Lagos State Government will not put an end to the menace. Rather than apportioning blames and even compulsorily acquiring the sites of collapsed buildings, including imprisonment of the builders, planning approving officers, and construction engineers, recommendations in this paper should be adopted for policy formulation and implementation.

\section{REFERENCES}

Adediji, B. (2006) Incessant building collapse: estate surveyors and valuers' roles, responsibility and liability, Paper delivered at the CPD Seminar organized by Lagos State Branch of the Nigerian Institution of Estate Surveyors \& Valuers, $30^{\text {th }}$ August, 2006.

Ahmad, A. G. (2004) Understanding common building defects - the dilapidation survey report, Majalah Akitek, 16(1), pp. 19-21.

Akinpelu, J. A. (2002) The need for code of conduct, building regulations and by-laws for the building industry in Nigeria, The Professional Builder, Journal of the Nigerian Institute of Building, pp. 11-14.

Ayininuola, G. M. and Olalusi, O. O. (2004) Assessment of building failures in Nigeria: Lagos and Ibadan case study, African Journal of Science and Technology, 5(1), pp. 73-78.

Bažant, Z. P. and Verdure, M. (2006) Mechanics of progressive collapse: learning from World Trade Center and building demolitions, Report No. 06-06/C605T, Department of Civil and Environmental Engineering, Northwestern University, Evanston, Illinois 60208, USA.
Available at: http://www.debunking911.com/ ProgressiveCollapseWTC-6-23-2006.pdf [accessed 21 December 2009]

Bažant, Z. P. and Zhou, Y. (2002) Why did the World Trade Center collapse?-Simple analysis, Journal of Engineering Mechanics-ASCE, 128(1), pp. 2-6. doi:10.1061/(ASCE)0733-9399(2002)128:1(2)

Binda, L., Gatti, G., Mangano, G., Poggi, C. and Landriani, G. S. (1992) The collapse of the Civic Tower of Pavia: a survey of the materials and structure, Masonry International, 6(1), pp. 11-20.

Bohr, B (2006) Map of the Local Government Areas of Lagos [Online] Available at: http:// de.wikipedia.org/wiki/Benutzer:Bohr [accessed 26 May 2008]

Merritt, F. and Ambrose, J. (1989) Building engineering and systems design, $2^{\text {nd }}$ Edition. New York: Springer.

Global Corruption Report (2005) Corruption in practice [Online] Transparency International. Available at: http://www.transparency.org/ publications/gcr/gcr_2005\#download [accessed 10 February 2010]

Hall, G. T. (1984) Revision notes on building maintenance and adaptation. Butterworths, England.

Heinle, E. and Leonhardt, F. (1996) Towers: a historical survey. New York: Butterworth-Heinemann.

Islam, S. (2008) 17 killed in India building collapse [Online] AHN. Available at: http://www. allheadlinenews.com/articles/7010986413 [accessed 26 May 2008]

Ismayilov, R. (2007) Azerbaijan: building collapse exposes "chaos" in Baku's urban planning [Online] The Open Society Institute. Available at: http://www.eurasianet.org/departments/ insight/articles/eav090607.shtml [accessed 26 May 2008]

Levy, M. and Salvadori, M. (1992) Why buildings fall down? New York: W.W. Norton \& Co.

Lewis, J. (2003) Housing construction in earthquake-prone places: perspectives, priorities and projections for development, The Australian Journal of Emergency Management, 18(2), pp. 35-44.

Oni, A. O. (2008) An empirical study of the Lagos State Rent Edict of 1997, Journal of the Nige- 
rian Institution of Estate Surveyors and Valuers, 31(1), pp. 20-32.

Oni, A. O. (2009) Arterial road network and commercial property values in Ikeja, Nigeria. Unpublished Ph.D. Thesis, Department of Estate Management, Covenant University, Ota, Nigeria.

Oyewande, B. (1992) A research for quality in the construction industry, Builder's Magazine, June/July Ed., Lagos.

Ozerdem, A. (1999) Tiles, taps and earthquake-proofing: lessons for disaster management in Turkey, Environment and Urbanisation, 11(2), pp. 177-180. doi:10.1177/095624789901100215

Pearson, C. and Delatte, N. (2005) Ronan Point apartment tower collapse and its effect on building codes, Journal of Performance of Constructed Facilities, 19(2), pp. 172-177. doi:10.1061/(ASCE)0887-3828(2005)19:2(172)
Richards, R. L. (2002) Leading the way in concrete repair and protection technology, Concrete Repair Association, Costa Rica, pp. 1.

Roddis, W. M. K. (1993) Structural failures and engineering ethics, Journal of Structural Engineering-ASCE, 119(5), pp. 1539-1555. doi:10.1061/(ASCE)0733-9445(1993)119:5(1539)

Wardhana, K. and Hadipriono, F. C. (2003) Study of recent building failures in the United States, Journal of Performance of Constructed Facilities, 17(3), pp. 151-158. doi:10.1061/(ASCE)0887-3828(2003)17:3(151)

Yussuf, S. A. (2006) Planning strategies for stemming building collapse in Lagos, Paper presented at CPD Seminar organized by the Nigerian Institution of Estate Surveyors \& Valuers Lagos State Branch on $30^{\text {th }}$ August 2006.

\section{SANTRAUKA}

\section{PASTATŲ GRIUVIMO ATVEJŲ DAŽNUMO LAGOSE (NIGERIJA) ANALIZĖ}

\section{Ayotunde Olawande ONI}

Lagose griūvantys pastatai kelia nerimą gyventojams, vystytojams ir vyriausybei. Šiame tyrime nagrinëjamas pastatu griuvumo dažnumas Lagose per trisdešimt metų. Atlikta laiko eilučių analizè, siekiant nustatyti buvusius atvejus ir numatyti būsimų atvejų tendencijas. Be to, siekiant nustatyti, dèl kokiu priežasčių tiriamoje teritorijoje griūva pastatai, buvo pasirinktas išvadu procesas, pagrịstas ankstesnių atvejų tyrimo ataskaitomis. Erdvinė griuvimų analizė parode didelę koncentraciją pelkètose vietovėse, kurios anksčiau buvo melioruotos. Be kitų dalykų, tyrime rekomenduojama atlikti išsamų paveiktų vietoviu geofizinių savybių tyrima, siekiant rasti ilgalaiki sprendima, kaip išvengti šios grèsmės. 